\title{
The mutational landscape of chronic neutrophilic leukemia: Case report and literature review
}

\author{
Stephen E Langabeer1* and Ezzat Elhassadi2 \\ ${ }^{1}$ Cancer Molecular Diagnostics, St. James's Hospital, Dublin, Ireland \\ ${ }^{2}$ Department of Haematology, University Hospital Waterford, Waterford, Ireland
}

\section{Introduction}

Chronic neutrophilic leukemia (CNL) is a rare myeloproliferative neoplasm characterised by mature neutrophil leucocytosis, a hypercellular bone marrow with minimal dysplasia and myeloblasts, and the presence of CSF3R mutations [1-2]. Historically, the overall survival of $\mathrm{CNL}$ patients is poor with transformation to blast crisis frequent [3]. The discovery of CSF3R mutations in this neoplasm, most frequently the T618I, has provided a rationale for targeted therapy with inhibitors of the JAK-STAT or SRC signalling pathways [4] borne out by some in vivo evidence $[5,6]$. However, some case reports suggest that a clinical response to JAK2 inhibition may be dependent on the underlying mutational heterogeneity [7]. Despite these advances, allogeneic stem cell transplantation (ASCT) remains the only potentially curative option but is limited to those eligible patients [8]. Therefore in order to stratify CNL patients into those who may benefit from JAK2 inhibitors or ASCT, evaluation of the individual patients' mutational landscape may be increasingly necessary. Here we describe the results of a targeted next-generation sequencing panel in a patient presenting with suspected $\mathrm{CNL}$ and summarize the current literature regarding the mutational landscape of this malignancy.

\section{Case report}

A 54 year-old male presented with a history of weight loss, a white cell count of $78.8 \times 10^{9} / \mathrm{L}$ (of which neutrophils $68.3 \times 10^{9} / \mathrm{L}$ ), hemoglobin of $13.0 \mathrm{~g} / \mathrm{dL}$ and platelets of $211 \times 10^{9} / \mathrm{L}$. The bone marrow aspirate was markedly hypercellular with increased, dysplastic megakaryocytes noted. There was a marked myeloid hyperplasia, left shift, prominent eosinophils with less than $5 \%$ myeloblasts, consistent with a myeloproliferative neoplasm (Figure 1). The patient had a normal karyotype, no PDGFRA or PDGFRB rearrangements, no $B C R$ $A B L 1$ transcripts and the JAK2 V617F mutation was not detected, all consistent with a diagnosis of CNL. Mutations were sought in diagnostic peripheral blood DNA using a targeted next-generation sequencing panel incorporating 27 genes commonly mutated in myeloid malignancies. This approach revealed mutations in CSF3R (p.Thr618Ile), ASXL1 (p.Val807Phefs ${ }^{\star} 11$ ), SETBP1 (p.Gly870Ser), and SRSF2 (p.Pro95Leu) consistent with a diagnosis of CNL (Table 1).

The patient commenced hydroxyurea (1g every third day and $500 \mathrm{mg}$ alternate days), then ruxolitinib (10mg bd) and allopurinol (300mg daily) but has not achieved any appreciable reduction in white cell count after eight months. The patient has continual weight loss and has subsequently developed splenomegaly $(20 \mathrm{~cm})$. In the absence of any sibling donors, a non-related donor search has been activated.

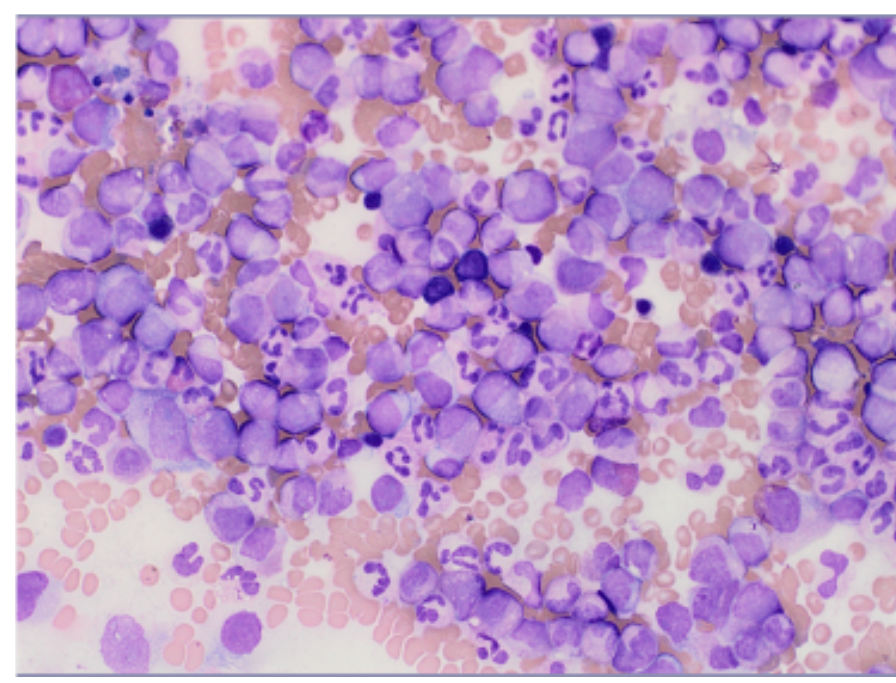

Figure 1. Diagnostic bone marrow morphology demonstrating hypercellularity with marked myeloid expansion and left shift

\section{Discussion}

CSF3R mutations represent a recurrent element of CNL cases with detection now a diagnostic criterion [1]. In addition to the CSF3R T618I, this patient also evidence of mutations in SETBP1, ASXL1, and SRSF2 reflecting the underlying, complex clonal architecture. A review of previous molecular profiling studies [9-13] has shown that these mutations are common co-operating events in CNL patients (Table 1). The clinical implications of these additional mutations remains poorly understood, largely due to the small number of CNL patients studied. As in the case described herein, a SETBP1 mutation has been previously associated with ruxolitinib unresponsiveness [7] with ASXL1 mutations a poor prognostic factor [10] hence the decision to proceed with an unrelated donor search with a view to ASCT.

This case further underscores the complexity of the mutational landscape of patients with CNL. Targeted NGS profiling is likely to play

${ }^{\star}$ Correspondence to: Stephen E Langabeer, Cancer Molecular Diagnostics, Central Pathology Laboratory, St. James's Hospital, Dublin D08 E9P6, Ireland, Tel: +353 1 4103576; Fax: +353 1 4103513; E-mail: slangabeer@stjames.ie

Key words: chronic neutrophilic leukemia, mutations, targeted therapy

Received: September 01, 2018; Accepted: October 26, 2018; Published: November 01, 2018 
Table 1. Summary of targeted sequencing studies in chronic neutrophilic leukemia depicting number of cases with the relevant mutated genes

\begin{tabular}{|c|c|c|c|c|c|c|c|c|c|}
\hline & \multicolumn{2}{|c|}{ CSF3R } & SETBP1 & SRSF2 & TET2 & ASXL1 & CALR & CBL & NRAS \\
\hline Meggendorfer, et al. [9] & $8 / 14$ & $(57 \%)$ & $2 / 14(14 \%)$ & $3 / 14(21 \%)$ & $4 / 14(29 \%)$ & $4 / 6(67 \%)$ & & & \\
\hline Elliott, et al. [10] & $14 / 14$ & $(100 \%)$ & $5 / 13(38 \%)$ & & & $8 / 14(57 \%)$ & $1 / 13(8 \%)$ & & \\
\hline Cui, et al. [11] & \multicolumn{2}{|c|}{$8 / 8(100 \%)$} & $6 / 8(75 \%)$ & & & & $1 / 8(12 \%)$ & & \\
\hline Ouyang, et al. [12] & $8 / 10$ & $(80 \%)$ & $1 / 10(10 \%)$ & & & & & & \\
\hline Langabeer, et al. [13] & \multicolumn{2}{|c|}{$4 / 4(100 \%)$} & $3 / 4(75 \%)$ & $4 / 4(100 \%)$ & & & & $1 / 4(25 \%)$ & $1 / 4(25 \%)$ \\
\hline This case & & 1 & 1 & 1 & & 1 & & & \\
\hline
\end{tabular}

an integral role in the future prognostic and therapeutic stratification of CNL patients.

\section{Authorship and contributorship}

SEL managed molecular studies. EE provided patient care and clinical information. Both authors contributed to manuscript preparation and approved the final submission.

\section{Acknowledgements}

None

\section{Funding information}

None

\section{Competing interests}

The authors declare no competing interests.

\section{References}

1. Arber DA, Orazi A, Hasserjian R, Thiele J, Borowitz MJ, et al. (2016) The 2016 revision to the World Health Organization classification of myeloid neoplasms and acute leukemia. Blood 127: 2391-2405.

2. Pardanani A, Lasho TL, Laborde RR, Elliot M, Hanson CA, et al. (2013) CSF3R T618I is a highly prevalent and specific mutation in chronic neutrophilic leukemia. Leukemia 27: 1870-1873.

3. Elliott MA, Tefferi A (2018) Chronic neutrophilic leukemia: 2018 update on diagnosis, molecular genetics and management. Am J Hematol 93: 578-587.
4. Maxson JE Gotlib J, Pollyea DA, Fleischman AG, Agarwal A, et al. (2013) Oncogenic CSF3R mutations in chronic neutrophilic leukemia and atypical CML. New England Journal of Medicine 368: 1781-1790.

5. Stahl M, Xu ML, Steensma DP, Rampal R, Much M, et al. (2016) Clinical response to ruxolitinib in CSF3R T618-mutated chronic neutrophilic leukemia. Annals of Hematology 95: 1197-1200.

6. Gunawan AS, McLornan DP, Wilkins B, Waghorn K, Hoade Y, et al. (2017) Ruxolitinib, a potent JAK1/JAK2 inhibitor, induces temporary reductions in the allelic burden of concurrent CSF3R mutations in chronic neutrophilic leukemia. Haematologica 102: e238-e240.

7. Ammantuna E, Eefting M, van Lom K, Kavelaars FG, Valk PJ, et al. (2015) Atypical chronic myeloid leukemia with concomitant CSF3R T618I and SETBP1 mutations unresponsive to the JAK2 inhibitor ruxolitinib. Annals of Hematology 94: 879-880.

8. Szuber N, Tefferi A (2018) Chronic neutrophilic leukemia: new science and new diagnostic criteria. Blood Cancer Journal 8: 19.

9. Meggendorfer M, Haferlach T, Alpermann T, Jeromin S, Haferlach C. et al. (2014) Specific molecular mutation patterns delineate chronic neutrophilic leukemia, atypical chronic myeloid leukemia, and chronic myelomonocytic leukemia. Haematologica 99: e244-e246.

10. Elliott MA, Pardanani A, Hanson CA, Lasho TL, Finke CM, et al. (2015) ASXL1 mutations are frequent and prognostically detrimental in CSF3R-mutated chronic neutrophilic leukemia. Am J Hematol 90: 653-656.

11. Cui Y, Li B, Gale RP, Jiang Q, Xu Z, et al. (2014) CSF3R, SETBP1 and CALR mutations in chronic neutrophilic leukemia. J Hematol Oncol 7: 77. [Crossref]

12. Ouyang Y, Qiao C, Chen Y, Zhang SJ (2017) Clinical significance of CSF3R, SRSF2 and SETBP1 mutations in chronic neutrophilic leukemia and chronic myelomonocytic leukemia. Oncotarget 8: 20834-20841.

13. Langabeer SE, Haslam K, Kelly J, Quinn J, Morrell R, et al. (2018) Targeted nextgeneration sequencing identifies clinically relevant mutations in patients with chronic neutrophilic leukemia at diagnosis and blast crisis. Clin Transl Oncol 20: 420-423.

Copyright: $@ 2018$ Langabeer SE. This is an open-access article distributed under the terms of the Creative Commons Attribution License, which permits unrestricted use, distribution, and reproduction in any medium, provided the original author and source are credited. 